\title{
GRAPH REGULARIZED COUPLED SPECTRAL UNMIXING FOR CHANGE DETECTION
}

\author{
Naoto Yokoya ${ }^{1}$ and Xiaoxiang $Z h u^{2,3}$ \\ ${ }^{1}$ Department of Advanced Interdisciplinary Studies, University of Tokyo, Japan \\ ${ }^{2}$ Remote Sensing Technology Institute (IMF), German Aerospace Center (DLR), Germany \\ ${ }^{3}$ Chair of Remote Sensing Technology, Technische Universität München (TUM), Germany
}

\begin{abstract}
This paper presents a methodology of coupled spectral unmixing for multitemporal hyperspectral data analysis. Coupled spectral unmixing simultaneously extracts the sets of spectral signatures of endmembers and respective abundance maps from multiple spectral images with differences in observation conditions and sensor characteristics. The problem is formulated in the framework of coupled nonnegative matrix factorization. A graph regularization that reflects spectral correlation between two images on abundance fractions is introduced into the optimization of coupled spectral unmixing to consider temporal changes of the earth's surface. An alternating optimization algorithm is investigated using the method of Lagrange multipliers to guarantee a stable convergence. The proposed method was applied to dual-temporal Hyperion images taken over the Fukushima Daiichi nuclear power plant. Experimental results showed that the proposed method can extract essential information on the earth's surface in a data-driven manner beyond multitemporal data modality.
\end{abstract}

Index Terms - Coupled spectral unmixing, graph regularization, change detection, multitemporal analysis

\section{INTRODUCTION}

Change detection has been an important task in multitemporal remote sensing data analysis. Spaceborne hyperspectral imaging systems are promising for monitoring and characterising the earth's environment on a global scale owing to its advanced ability for accurate identification and classification. Several spaceborne hyperspectral imaging missions, such as the environmental mapping and analysis program (EnMAP) and hyperspectral imager suite (HISUI), are going to be operational in the near future. An analysis of multitemporal hyperspectral data is expected to be useful for understanding dynamic changes of the earth's surface in details for a wide range of applications, for example, disaster management, forest monitoring and precision agriculture. However, it raises the demand for sophisticated algorithms to deal with the high dimensional nature of the data as well as multitemporal data

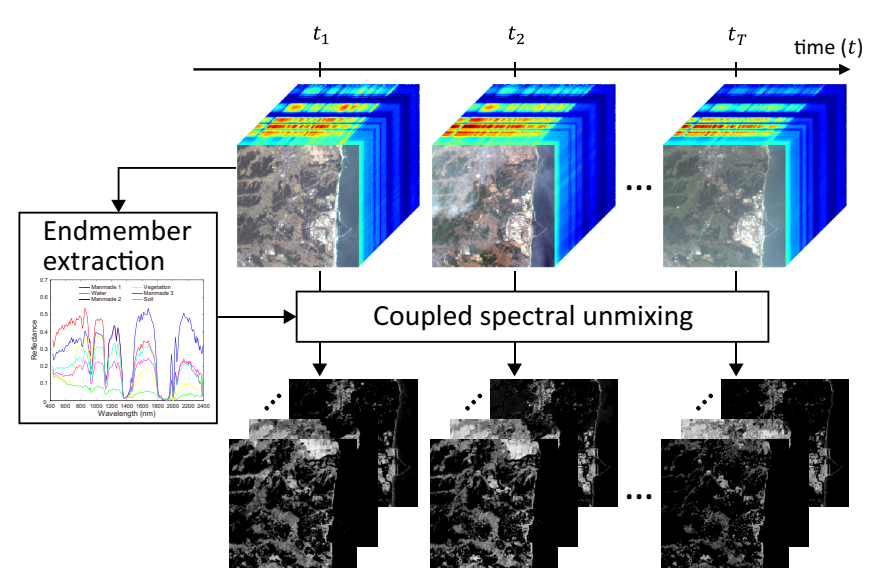

Fig. 1. Concept of coupled spectral unmixing.

modality due to the effect of atmospheric conditions, sensor calibration and ground conditions, etc.

Supervised classification methods have been commonly used for class-specific change detection using multitemporal spectral images [1]; however, they usually require a large amount of training samples to obtain an accurate classifier, which often cost high in terms of human and economic resources. Spectral unmixing has been actively developed as a key technology in hyperspectral image processing to retrieve spectral signatures of endmembers and their fractional abundance maps in a data-driven manner. When applied to multitemporal data analysis, spectral unmixing is expected to be useful not only for class-specific change detection [2] but also for within-class change detection. However, few studies have been carried out so far on multitemporal spectral unmixing that uses spectral correlation between multitemporal spectral images to improve the unmixing accuracy and robustness against multitemporal data mismatch.

Multitemporal coupled spectral unmixing is defined as a process that simultaneously estimates the sets of spectral signatures of endmembers and abundance maps from multitemporal hyperspectral data in a data-driven manner. The benefits of multitemporal hyperspectral image analysis based on spectral unmixing are threefold: 1) data-driven information extraction of the earth's surface owing to rich spatial-spectral- 
temporal data; 2) class-specific change detection; 3) withinclass change detection. This paper presents a methodology of multitemporal coupled spectral unmixing for land-cover change detection. Coupled spectral unmixing for multitemporal hyperspectral data is formulated in the framework of coupled nonnegative matrix factorization [3] using graph regularization on spectral correlation between multitemporal data. Experiments are conducted on the analysis of dual-temporal Hyperion images taken over the Fukushima Daiichi nuclear power plant in 2012 and 2014 to visualize the changes of landcover classes.

\section{METHODOLOGY}

\subsection{Spectral unmixing based on nonnegative matrix fac- torization}

A linear spectral mixture model is commonly used for unmixing problems owing to its physical effectiveness and mathematical simplicity. A spectrum at each pixel is assumed to be a linear combination of several endmember spectra. Therefore, a hyperspectral image $\mathbf{X} \in \mathbb{R}^{B \times P}$, with $B$ bands and $P$ pixels, is formulated as

$$
\mathbf{X}=\mathbf{E A}^{T}+\mathbf{N}
$$

where $\mathbf{E} \in \mathbb{R}^{B \times M}$ is the spectral signature matrix, with $M$ being the number of endmembers, $\mathbf{A} \in \mathbb{R}^{P \times M}$ is the abundance matrix, $\mathbf{N} \in \mathbb{R}^{B \times P}$ is the residual, and the operator ()$^{T}$ denotes the transposition operation. Researchers have studied many models and algorithms based on geometrical, statistical, and sparse regression-based approaches searching for robust, stable, tractable, and accurate unmixing [4].

Over the last decade, nonnegative matrix factorization (NMF) algorithms [5, 6] have emerged as useful unmixing methods because they overcome the difficulty of absence of pure pixels with straightforward implementation and mathematical flexibility to consider several constraints. Lu et al. presented a graph regularized $L_{1 / 2}-\mathrm{NMF}$ (GLNMF) that takes into account sparse characteristic and the intrinsic manifold structure of the data [7]. With a matrix $\mathbf{W} \in \mathbb{R}^{P \times P}$ representing the weight matrix of the graph, the optimization is given by

$$
\begin{gathered}
\min _{\mathbf{E}, \mathbf{A}}\left\|\mathbf{X}-\mathbf{E} \mathbf{A}^{T}\right\|_{F}^{2}+\alpha \sum_{j=1}^{P} \sum_{m=1}^{M} \mathbf{A}_{j m}^{1 / 2} \\
+\beta \sum_{j, l=1}^{P}\left\|\mathbf{a}_{j}-\mathbf{a}_{l}\right\|_{2}^{2} \mathbf{W}_{j l} \\
\text { s.t. } \mathbf{E} \succeq 0, \mathbf{A} \succeq 0, \sum_{m=1}^{M} \mathbf{A}_{j m}=1
\end{gathered}
$$

where $\mathbf{a}_{j}=\left[a_{j 1}, \ldots, a_{j M}\right]^{T},\|\cdot\|_{F}$ denotes the Frobenius norm, and the symbol $\succeq$ denotes inequality component-wise.
The following update rules can be derived by the method of Lagrange multipliers

$$
\begin{array}{cc}
\mathbf{E}_{i m} \leftarrow & \mathbf{E}_{i m} \frac{(\mathbf{X A})_{i m}}{\left(\mathbf{E} \mathbf{A}^{T} \mathbf{A}\right)_{i m}} \\
\mathbf{A}_{j m} \leftarrow \quad \mathbf{A}_{j m} \frac{\left(\mathbf{X}^{T} \mathbf{E}+\beta \mathbf{W A}\right)_{j m}}{\left(\mathbf{A E}^{T} \mathbf{E}+\frac{\alpha}{2} \mathbf{A}^{1 / 2}+\beta \mathbf{D A}\right)_{j m}}
\end{array}
$$

$\mathbf{D} \in \mathbb{R}^{P \times P}$ denotes a diagonal matrix whose entries are column sums of $\mathbf{W}$. There are many choices to define the weight matrix $\mathbf{W}$ on the graph, for example, $0-1$, heat kernel, and dot-product weighting. When both spatial and spectral distances are considered to construct the graph, each element of $\mathbf{W}$ is given by

$$
\mathbf{W}_{j l}=e^{\left(-\frac{d(j, l)}{\sigma_{d}}-\frac{\left\|\mathbf{x}_{j}-\mathbf{x}_{l}\right\|_{2}^{2}}{\sigma_{s}}\right)}
$$

where $d(j, l)$ denotes the Euclidean distance between the $j^{\text {th }}$ and its neighboring $l^{\text {th }}$ pixel, and $\sigma_{d}$ and $\sigma_{s}$ are the parameters for spatial and spectra distances, respectively.

\subsection{Graph-regularized coupled sparse nonnegative ma- trix factorization}

We extend GLNMF to multitemporal spectral unmixing by integrating the graph regularization on the temporal changes of spectra into two unmixing problems in a coupled basis. We assume that when the spectrum has changed, the abundance fractions have also changed, and vice versa. In this paper, we consider coupled spectral unmixing of two spectral images denoted by $\mathbf{X}_{1} \in \mathbb{R}^{B_{1} \times P}$ and $\mathbf{X}_{2} \in \mathbb{R}^{B_{2} \times P}$. The two images are geometrically co-registered with the same ground sampling distance but the band numbers can be different to use multiple sensors. Consider a weight matrix $\mathbf{W}_{t} \in$ $\mathbb{R}^{P \times P}$ with each element $\mathbf{W}_{t j l}$ corresponds to the similarity between $\mathbf{X}_{1 j}$ and $\mathbf{X}_{2 l}$. Graph-regularized coupled sparse nonnegative matrix factorization (GCSNMF) can be formulated as

$$
\begin{aligned}
& \min _{\mathbf{E}_{1}, \mathbf{A}_{1}, \mathbf{E}_{2}, \mathbf{A}_{2}}\left\|\mathbf{X}_{1}-\mathbf{E}_{1} \mathbf{A}_{1}^{T}\right\|_{F}^{2}+\left\|\mathbf{X}_{2}-\mathbf{E}_{2} \mathbf{A}_{2}^{T}\right\|_{F}^{2} \\
& +\alpha \sum_{j=1}^{P} \sum_{m=1}^{M}\left(\mathbf{A}_{1_{j m}}^{1 / 2}+\mathbf{A}_{2}{ }_{j m}^{1 / 2}\right) \\
& +\beta \sum_{j, l=1}^{P}\left(\left\|\mathbf{a}_{1 j}-\mathbf{a}_{1 l}\right\|_{2}^{2} \mathbf{W}_{1 j l}+\left\|\mathbf{a}_{2 j}-\mathbf{a}_{2 l}\right\|_{2}^{2} \mathbf{W}_{2 j l}\right) \\
& +\gamma \sum_{j, l=1}^{P}\left\|\mathbf{a}_{1 j}-\mathbf{a}_{2 l}\right\|_{2}^{2} \mathbf{W}_{t j l}+\delta\left\|\mathbf{R E}_{1}-\mathbf{E}_{2}\right\|_{F}^{2} \\
& \text { s.t. } \mathbf{E}_{1} \succeq 0, \mathbf{A}_{1} \succeq 0, \mathbf{E}_{2} \succeq 0, \mathbf{A}_{2} \succeq 0, \sum_{m} \mathbf{A}_{1 j m}=1, \sum_{m} \mathbf{A}_{2 j m}=1
\end{aligned}
$$

where $\mathbf{E}_{1} \in \mathbb{R}^{B_{1} \times M_{1}}$ and $\mathbf{A}_{1} \in \mathbb{R}^{P \times M_{1}}$ are the signature and abundance matrices of the first data $\mathbf{X}_{1}, \mathbf{E}_{2} \in \mathbb{R}^{B_{2} \times M_{2}}$ and $\mathbf{A}_{2} \in \mathbb{R}^{P \times M_{2}}$ are those of the second data $\mathbf{X}_{2}$, and $\mathbf{R} \in \mathbb{R}^{B_{2} \times B_{1}}$ is the relative spectral response function. In addition to the sparsity constraint and the spatial-spectral graph regularization for each unmixing, the term with the parameter $\delta$ is the temporal graph regularization. The update rules can 
be obtained by the method of Lagrange multipliers

$$
\begin{aligned}
& \mathbf{E}_{1 i m} \leftarrow \\
& \mathbf{A}_{1 j m} \leftarrow \quad \mathbf{E}_{1 i m} \frac{\left(\mathbf{X}_{1} \mathbf{A}_{1}+\delta \mathbf{R}^{T} \mathbf{E}_{2}\right)_{i m}}{\left(\mathbf{E}_{1} \mathbf{A}_{1}^{T} \mathbf{A}_{1}+\delta \mathbf{R}^{T} \mathbf{R} \mathbf{E}_{1}\right)_{i m}} \frac{\left(\mathbf{X}_{1}^{T} \mathbf{E}_{1}+\beta \mathbf{W}_{1} \mathbf{A}_{1}+\gamma \mathbf{W}_{t} \mathbf{A}_{2}\right)_{j m}}{\left(\mathbf{A}_{1} \mathbf{E}_{1}^{T} \mathbf{E}_{1}+\frac{\alpha}{2} \mathbf{A}_{1}^{1 / 2}+\beta \mathbf{D}_{1} \mathbf{A}_{1}+\gamma \mathbf{D}_{\mathbf{t} 1} \mathbf{A}_{1}\right)_{j m}} \\
& \mathbf{E}_{2 k m} \leftarrow \\
& \mathbf{E}_{2 k m} \leftarrow \frac{\left(\mathbf{X}_{2} \mathbf{A}_{2}+\delta \mathbf{R} \mathbf{E}_{1}\right)_{k m}}{\left(\mathbf{E}_{2} \mathbf{A}_{2}^{T} \mathbf{A}_{2}+\delta \mathbf{E}_{2}\right)_{k m}} \\
& \mathbf{A}_{2 j m} \leftarrow \\
& \mathbf{A}_{2 j m} \frac{\left(\mathbf{X}_{2}^{T} \mathbf{E}_{2}+\beta \mathbf{W}_{2} \mathbf{A}_{2}+\gamma \mathbf{W}_{t}^{T} \mathbf{A}_{1}\right)_{j m}}{\left(\mathbf{A}_{2} \mathbf{E}_{2}^{T} \mathbf{E}_{2}+\frac{\alpha}{2} \mathbf{A}_{2}^{1 / 2}+\beta \mathbf{D}_{2} \mathbf{A}_{2}+\gamma \mathbf{D}_{\mathbf{t} 2} \mathbf{A}_{2}\right)_{j m}}
\end{aligned}
$$

where $\mathbf{D}_{t 1}$ and $\mathbf{D}_{t 2}$ denote diagonal matrices whose entries are column and row sums of $\mathbf{W}_{t}$, respectively. $\mathbf{W}_{t}$ is designed as a diagonal matrix using the spectral angle distance for the spectral similarity measurement. The abundance sumto-one constraint is implemented using a method given in [9].

After the initialization of all the matrices, they are alternately optimized by Equations (7)-(10). In this paper, we present a specific implementation of GCSNMF. Firstly, two images are obtained by the same sensor, and thus $\delta$ is set to 0 . Secondly, the two images mostly include the same endmembers with spectral mismatch. Thirdly, the data is assumed to contain all pure pixels of analyzed materials.

The data matrix $\mathbf{X}_{1}$ is considered as the reference data and the endmember matrix $\mathbf{E}_{1}$ is estimated by vertex component analysis (VCA) [8]. The endmember matrix $\mathbf{E}_{2}$ is initialized as $\left[\mathbf{E}_{1} \mathbf{r}\right]$, where $\mathbf{r} \in \mathbf{R}^{B}$ is a mean vector of the residual between the two images, i.e., $\mathbf{X}_{2}-\mathbf{X}_{1}$, with all negative components replaced by a very small positive value. The abundance matrices $\mathbf{A}_{1}$ and $\mathbf{A}_{2}$ are initialized by the fully constrained least-squares (FCLS) method [9]. During the optimization of GCSNMF, $\mathbf{A}_{1}$ and $\mathbf{A}_{2}$ are optimized by Equations (8) (10), respectively. For the spectral signatures, only the last column vector of $\mathbf{E}_{2}$ is updated as a virtual endmember signature that explains spectral mismatch between the two images. Classspecific changes of land cover can be visualized by comparing multitemporal abundance maps. All the parameters in the cost function are empirically set taking a balance of the regularization terms.

\section{EXPERIMENT}

The proposed method is applied to dual-temporal Hyperion images taken over the Fukushima Daiichi nuclear power plant on 29 April 2012 and 25 May 2014. The hyperspectral data cubes with composite RGB images are shown in Fig. 2. Atsensor reflectance data that include atmospheric effects are used with 197 effective bands. The first image taken on 29 April 2012 is used for the reference to extract endmembers. In this experiment, totally six endmembers are extracted by VCA and labeled by human as three types of man-made materials, vegetation, soil, and water. When applied to at-surface reflectance data, a spectral dictionary is useful to make this labeling process automatic. The proposed coupled spectral
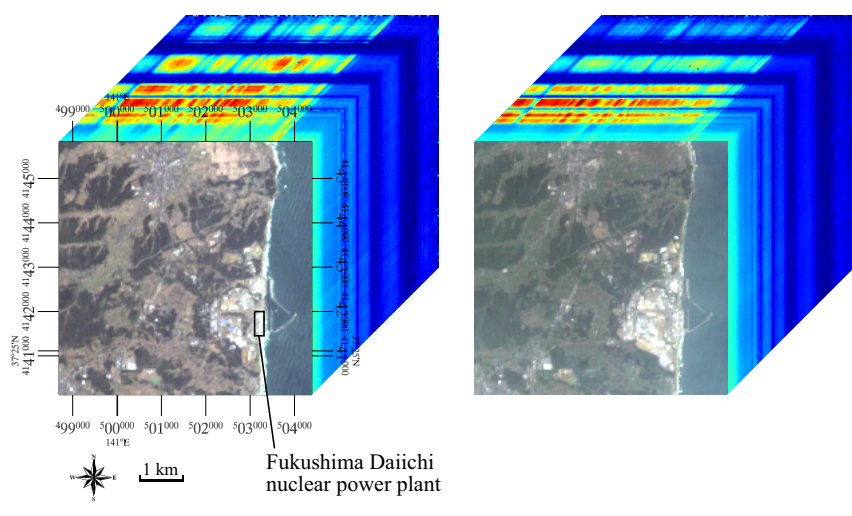

Fig. 2. Hyperion images taken over the Fukushima Daiichi nuclear power plant on (left) 29 April 2012 and (right) 25 May 2014, respectively.

unmixing method is compared with the conventional individual spectral unmixing methods, i.e., FCLS and GLNMF. Note that the same endmember signatures are used for all methods.

Fig. 3(a) shows the unmixing results on the first image obtained by the three methods, whereas Fig. 3(b) shows those on the second image. The last row images are composite RGB images using abundance maps of man-made materials, vegetation, and soil for red, green, and blue, respectively. Fig. 3(c) shows the difference maps of abundances with RGB images visualizing class-specific changes. For example, magenta, green, orange, and purple indicate the changes from vegetation to man-made, from soil to vegetation, from soil to man-made, and from vegetation to soil, respectively. In the FCLS results, large abundance errors of water appear in the land area as a result of the strict sum-to-one constraint. In the results obtained by FCLS and GLNMF, there are too much abundance increase in vegetation caused by data mismatch due to atmospheric effects. The proposed method mitigated this error owing to the temporal constraint. In addition, the results obtained by the individual spectral unmixing methods show too much abundance increase of man-made materials in the Fukushima Daiichi nuclear power plant and the urban area located on the northwest part of the power plant, although they have just partly changed rather than largely. The proposed method shows stable and reasonable results on these areas. Accordingly, the proposed method seems to achieve the accurate spectral unmixing and its robustness against temporal data mismatch. In the RGB image of abundance difference obtained by GCSNMF, trees that have been cut down to increase the number of water storage tanks in and around the power plant can be clearly visualized as magenta and purple pixels. The increase of vegetation may be because of the growth of weeds owing to seasonal difference as well as the yearly expansion of the abandoned farmland in the restricted area. 


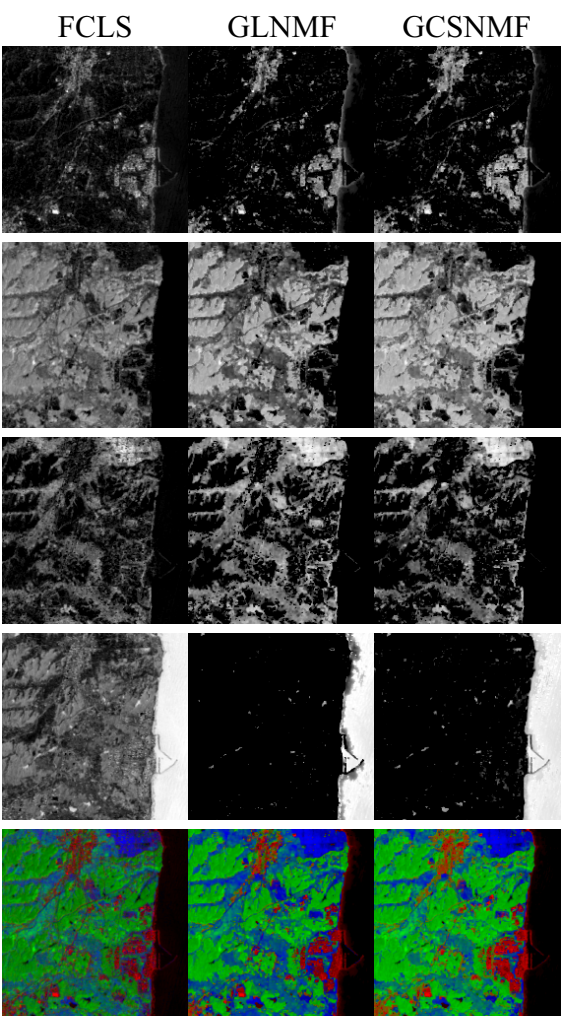

(a)

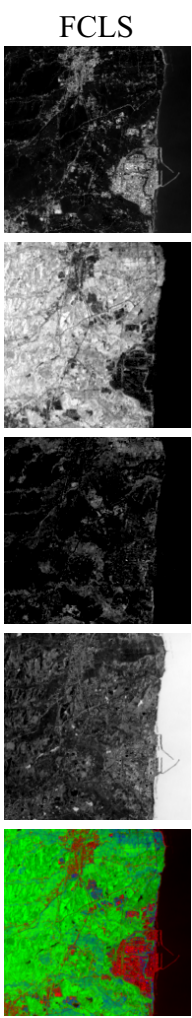

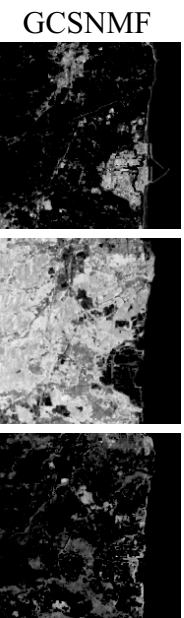
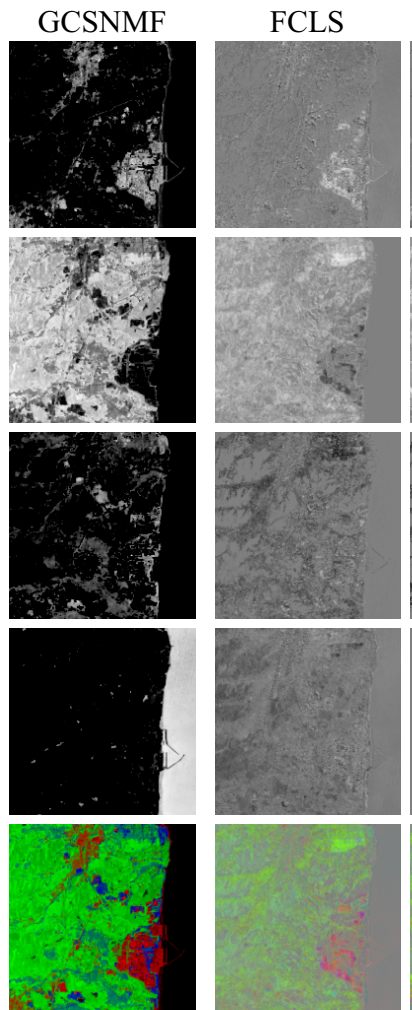

(b)

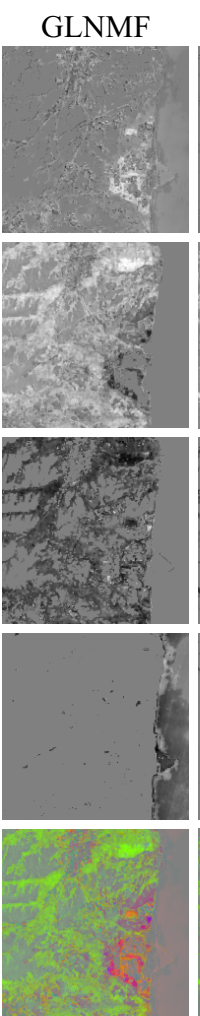

(c)

Fig. 3. Unmixing results of data acquired on (a) 29 April 2012 and (b) 25 May 2014, and (c) their difference images obtained by FCLS, GLNMMF, and GCSNMF from left to right. Abundance maps of man-made materials, vegetation, soil, and water, and composite RGB images using abundance maps of man-made materials, vegetation, and soil for red, green, and blue, respectively, are shown from top to bottom. The figure can be best viewed with $200 \%$ zoom.

\section{CONCLUSION}

This paper present a coupled spectral unmixing method for multitemporal hyperspectral data analysis. The proposed method introduces a graph regularization on spectral changes into the optimization of multitemporal spectral unmixing in a coupled basis in order to improve the unmixing accuracy and robustness against multitemporal data mismatch. The proposed method has been applied to dual-temporal Hyperion images taken over the Fukushima Daiichi nuclear power plant and successfully visualized class-specific changes of land cover. Coupled spectral unmixing is expected to be a powerful tool for multitemporal spectral data analysis enabling data-driven extraction of essential information on the earth's surface that cannot be obtained from a single image. Our future work includes investigation on numerical validation and application to multisensor data.

\section{REFERENCES}

[1] G. Camps-Valls, L. Gmez-Chova, J. Muoz-Mar, J. L. Rojo-lvarez, and M. Martnez-Ramn, "Kernel-based framework for multitemporal and multisource remote sensing data classification and change detection," IEEE Trans. Geosci. Remote Sens., vol. 46, no. 6, pp. 1822-1835, 2008.

[2] D. Lu, M. Batistella, and E. Moran, "Multitemporal spectral mixture analysis for Amazonian land-cover change detection," Can. J. Remote Sens., vol. 30, no. 1, pp. 87-100, 2004.

[3] N. Yokoya, T. Yairi, and A. Iwasaki, "Coupled nonnegative matrix factorization unmixing for hyperspectral and multispectral data fusion," IEEE Trans. Geosci. Remote Sens., vol. 50, no. 2, pp. 528-537, 2012.

[4] J. M. Bioucas-Dias, A. Plaza, N. Dobigeon, M. Parente, Q. Du, P. Gader, and J. Chanussot, "Hyperspectral unmixing overview: geometrical, statistical, and sparse regression-based approaches," IEEE J. Sel. Topics Appl. Earth Observ., vol. 5, no. 2, pp. 354-379, 2012.

[5] D. D. Lee and H. S. Seung, "Learning the parts of objects by nonnegative matrix factorization," Nature, vol. 401, pp. 788-791, 1999.

[6] D. Cai, X. He, J. Han, and T. S. Huang, "Graph regularized non-negative matrix factorization for data representation," IEEE Trans. Pattern Anal. Mach. Intell., vol. 33, no. 8, pp. 1548-1560, 2010.

[7] X. Lu, H. Wu, Y. Yuan, P. Yan, and X. Li, "Manifold regularized sparse NMF for hyperspectral unmixing," IEEE Trans. Geosci. Remote Sens., vol. 51, no. 5, pp. 2815-2826, 2013.

[8] J. M. P. Nascimento and J. M. B. Dias, "Vertex component analysis: A fast algorithm to unmix hyperspectral data," IEEE Trans. Geosci. Remote Sens., vol. 43, no. 4, pp. 898-910, 2005.

[9] D. C. Heinz and C.-I. Chang, "Fully constrained least squares linear spectral mixture analysis method for material quantification in hyperspectral imagery," IEEE Trans. Geosci. Remote Sens., vol. 39, no. 3, pp. 529-545, 2001. 